\title{
ANÁLISE ECONÔMICA DETERMINÍSTICA DA TERMINAÇÃO EM CONFINAMENTO DE NOVILHOS ABATIDOS COM DISTINTOS PESOS
}

\author{
PaUlo SANTANA PACHECO ${ }^{1}$, JoÃo Restle ${ }^{2}$, KAROLINE GOMES VALENÇA ${ }^{3}$, DANIEL \\ BATISTA LEMES ${ }^{3}$, FERNANDA REZER DE MENEZES ${ }^{4}$, GREICE KELly GOMES MACHADO ${ }^{3}$ \\ ${ }^{1}$ Professor, Dr. Universidade Federal de Santa Maria, Santa Maria, RS, Brasil. E-mail: pacheco.dz.ufsm@hotmail.com \\ ${ }^{2} \mathrm{PhD}$. Universidade Federal do Tocantins, Araguaína, TO, Brasil. \\ ${ }^{3}$ Discente em Zootecnia. Universidade Federal de Santa Maria, Santa Maria, RS, Brasil. \\ ${ }^{4}$ Discente em Estatística. Universidade Federal de Santa Maria, Santa Maria, RS, Brasil.
}

\begin{abstract}
O objetivo deste estudo foi o de avaliar a viabilidade econômica da terminação em confinamento de novilhos, por meio de indicadores financeiros de retorno e risco, via análise determinística, tomando como base valores de cotações médias praticadas no Rio Grande do Sul nos anos de 2004 a 2012. Foram utilizados 18 novilhos da raça Charolês, com idade média inicial de 30 meses e peso médio inicial de $297,0 \mathrm{~kg} \pm 11,5 \mathrm{~kg}$. Avaliaram-se três pesos de abate pré-determinados: 420, 460 e $500 \mathrm{~kg}$, sendo os pesos obtidos de 421, 461 e $495 \mathrm{~kg}$, respectivamente. O período de alimentação foi de 110 , 145 e 184 dias, respectivamente. Os custos variáveis representaram $98 \%$ do custo total, sendo que, destes, os mais representativos foram: compra do animal magro e
\end{abstract}

alimentação (volumoso +_concentrado). As estimativas dos indicadores financeiros para os pesos de abate de 421, 461 e $495 \mathrm{~kg}$ foram, respectivamente, de $\mathrm{R} \$-266,30$, $-323,49$ e $-417,18$ para margem bruta, $\mathrm{R} \$-289,70,-346,90$ e -440,59 para margem líquida, de R $\$-344,89,-419,93$ e $-536,24$ para lucro, de $\mathrm{R} \$-316,78,-381,92$ e $-483,67$ para valor presente líquido, de $0,85,0,83$ e 0,81 para índice benefício:custo, $-2,65 \%,-2,52 \%$ e $-2,58 \%$ para retorno adicional sobre o investimento, $-5,02 \%,-4,57 \%$ e $-4,56 \%$ para taxa interna de retorno (a.m.) e 7,05, 8,37 e 9,86 meses para payback descontado. Apesar de indicarem inviabilidade da terminação em qualquer peso de abate, menores pesos resultaram em menores perdas econômicas, tornando essa tecnologia de elevado risco econômico.

PALAVRAS-CHAVE: análise de investimentos, análise de risco, bovinos de corte, peso ideal de abate, sistema de terminação.

\section{ECONOMIC DETERMINISTIC ANALYSIS OF FEEDLOT-FINISHED STEERS SLAUGHTERED AT DIFFERENT WEIGHTS}

\section{ABSTRACT}

The aim of this study was to evaluate the economic viability of feedlot-finished steers through financial indicators of risk and return, via deterministic analysis, based on average values quotations practiced in Rio Grande do Sul state in the years 2004-2012. We used 18 Charolais steers with average initial age of 30 months and average initial weight of $297.0 \mathrm{~kg} \pm 11.5 \mathrm{~kg}$. We evaluated three predetermined slaughter weights: 420, 460 and
$500 \mathrm{~kg}$, being the weights obtained of 421,461 and $495 \mathrm{~kg}$, respectively. Variable costs represented $98 \%$ of total cost, and of these, the most representative were purchase of feeder cattle and food (forage + concentrate). The estimates of the financial indicators to slaughter weights of 421,461 and $495 \mathrm{~kg}$ were, respectively: $\mathrm{R} \$-266.30$, -323.49 and -417.18 for gross margin; $\mathrm{R} \$-289.70$, -440.59 and 346.90 for net margin; $\mathrm{R} \$-344.89,-419.93$ 
and -536.24 for profit; $\mathrm{R} \$-316.78,-381.92$ and -483.67 for net present value; $0.85,0.83$ and 0.81 for index benefit:cost, $-2.65 \%,-2.52 \%$ and $-2.58 \%$ for additional return on investment; $-5.02 \%,-4.57 \%$ and $-4.56 \%$ for the internal rate of return (a.m.); and 7.05, 8.37 and 9.86 months for discounted payback. Although these results indicate infeasibility of termination at any slaughter weight, lower weights resulted in lower economic losses, making this technology of high economic risk.

KEYWORDS: beef cattle, finishing system, ideal slaughter weight, investment analysis, risk analysis.

\section{INTRODUÇÃO}

Os sistemas de produção de bovinos de corte atualmente empregados no país caracterizam-se pela pouca intensificação, com uso predominante de pastagens. Conforme Millen et al. ${ }^{1}$, não mais do que $8 \%$ dos bovinos abatidos são oriundos de confinamento, tecnologia que apresenta algumas vantagens como a redução da idade de abate, produção de carne de melhor qualidade, aumento no desfrute reduzindo a ociosidade dos frigoríficos na entressafra, maior giro de capital, melhor aproveitamento das áreas de pastagens para outras categorias e elevada produção de adubo orgânico ${ }^{2}$. Porém, seu uso pode resultar em aumento significativo de investimentos. Em estudos avaliando a terminação de bovinos de corte com diferentes pesos de abate ${ }^{3}$, houve incrementos significativos no consumo de matéria seca, sendo esta variável diretamente relacionada aos custos com alimentação e, por consequência, ao custo total. Pacheco et al. ${ }^{4}$, Restle et al. ${ }^{5}$ e Missio et al. ${ }^{6}$ verificaram que os custos com alimentação representaram mais de $70 \%$ dos custos variáveis, desconsiderando o custo com aquisição do animal magro.

Com isso, quando se preconiza o incremento no peso de abate, ocorre aumento no período total de alimentação, justificando, com isso, a análise dos custos e receitas visando determinar qual peso de abate seria considerado ideal do ponto de vista econômico. Neste sentido, Souza et al. ${ }^{7}$ sugerem a análise conjunta de vários indicadores financeiros, resultando em informações mais consistentes da avaliação do risco e seu confronto com a possibilidade de retorno do investimento. Em estudos agronômicos, Kreuz et al. ${ }^{8}$, Kreuz e Souza ${ }^{9}$ e Kreuz et al. $^{10}$ demonstraram a maneira como a avaliação de vários indicadores financeiros pode auxiliar na tomada de decisão, inclusive diferenciando os mesmos em indicadores de retorno e de risco.

Objetivou-se com este estudo avaliar a viabilidade econômica da terminação em confinamento de novilhos abatidos com diferentes pesos, por meio de indicadores financeiros de retorno e risco, via análise determinística.

\section{MATERIAL E MÉTODOS}

Foram utilizados 18 novilhos, com idade média, ao início do experimento, de 30 meses e peso médio de $297,0 \mathrm{~kg} \pm 11,5 \mathrm{~kg}$. Os animais foram distribuídos ao acaso em lotes de três novilhos (dois lotes por tratamento) e alojados em piquetes a céu aberto, com $80 \mathrm{~m}^{2}$ de área, providos de cocho e bebedouro regulado por boia automática.

A dieta alimentar, contendo $12 \%$ de proteína bruta e $67,84 \%$ de nutrientes digestíveis totais, sendo constituída de cana-de-açúcar triturada, representando $43 \%$ da matéria seca (MS) e $57 \%$ de concentrado na MS. O concentrado era composto por sorgo em grão triturado $(63,0 \%)$, farelo de arroz desengordurado $(25,1 \%)$, farelo de soja $(8,1 \%)$, mistura mineral $(2,54 \%)$ e ureia $(1,26 \%)$. Os animais foram alimentados duas vezes ao dia, sendo $63 \%$ do alimento fornecido na parte da manhã e $37 \%$ na parte da tarde. O volumoso e o concentrado foram misturados no cocho no momento do fornecimento.

A fase de adaptação dos novilhos à dieta e ao manejo foi de 14 dias. Durante esse período, os animais foram tratados contra endo e ectoparasitas. Para controle da evolução do peso dos animais, foram realizadas pesagens periódicas, obedecendo a um jejum de sólidos de 14 horas.

Avaliaram-se três pesos de abate prédeterminados: 420, 460 e $500 \mathrm{~kg}$, sendo os pesos obtidos de 421, 461 e $495 \mathrm{~kg}$, respectivamente. Os pesos de abate foram considerados projetos de investimento mutuamente excludentes. Para estimativa dos custos de produção, bem como dos indicadores econômicos, considerou-se o método determinístico, que assume valores fixos (conhecidos) para os itens que compõem os custos e indicadores econômicos, tomando como base valores médios praticados no estado do Rio Grande do Sul nos anos de 2004 a 2012, das seguintes fontes: Conab - Companhia Nacional de Abastecimento; IEA - Instituto de Economia Agrícola de São Paulo; Emater/RS-ASCAR e Anualpec - Anuário da Pecuária Brasileira. Essas fontes foram escolhidas visando garantir a idoneidade das cotações utilizadas neste estudo.

Para classificação dos itens de custos, receitas e cálculos dos indicadores financeiros, foram 
utilizadas metodologias propostas por Matsunaga et al. ${ }^{11}$, Resende Filho et al. ${ }^{12}$, Lapponi ${ }^{13}$ e Souza et al. ${ }^{7}$.

Foram considerados os seguintes custos: compra do animal magro, alimentação (volumoso e concentrado), mão-de-obra, sanidade, depreciação e outras despesas operacionais. Todas as estimativas foram feitas por animal e por ano, deflacionados para 2012 pelo índice IGP-DI da Fundação Getúlio Vargas, associadas com as características de desempenho obtidas dos animais durante a fase de alimentação (peso, ganho de peso e consumo de matéria seca).

Para todos os pesos de abate avaliados, as cotações para definição do custo com compra do animal magro e receita com venda do animal gordo foram obtidas das médias para os meses de junho e novembro, respectivamente.

Os custos com instalações foram estimados para capacidade estática de 1.000 animais e vida útil de 10 anos. A depreciação (instalações) foi calculada para um horizonte de planejamento de um ano, mesmo período considerado no cálculo das depreciações de máquinas, implementos e equipamentos.

Os valores estimados para depreciações foram de R \$ 0,05 animal ${ }^{-1} \mathrm{dia}^{-1}$ com instalações e R \$ 0,04 animal $^{-1}$ dia $^{-1}$ com máquinas, implementos e equipamentos.

Os custos de oportunidade foram calculados considerando a taxa mínima de atratividade (TMA) de $0,58 \%$ a.m., correspondente ao rendimento da caderneta de poupança de 7,25 \% a.a., calculada através da seguinte equação: TMA (\% a.m.) $=(1+$ $7,25 \%$ a.a.) $1 / 12$ - 1 . O custo de oportunidade do capital investido foi obtido pelo somatório das despesas operacionais (compra animal magro, controle sanitário, alimentação volumoso + concentrado, mão-de-obra contratada/diarista + assistência técnica e outras), para o período correspondente ao número de meses estipulados para cada peso de abate. Para o custo de oportunidade da terra (referente apenas à área destinada para instalações do confinamento), determinou-se para cada animal o uso de 0,05 ha, com possibilidade de arrendamento anual da mesma pelo equivalente a 3\% do valor do hectare para cultura ( $\mathrm{R} \$ 7.388,30)$.

O controle sanitário consistiu em aplicação de produto para controle de endo e ectoparasitas (ivermectina 1\%), analgésico e anti-inflamatório, antibiótico e vacinas contra febre aftosa, botulismo e clostridiose, todos em dosagem por animal, conforme recomendações dos fabricantes.

O custo com alimentação foi obtido pelo produto entre o consumo total de volumoso e concentrado (em $\mathrm{kg}$ MS/animal) e seus respectivos custos/kg de MS. Os cálculos utilizados seguem os sugeridos por Pacheco et al. ${ }^{4}$.

Tabela 1 - Descrição dos itens de custos e receitas utilizados para cálculo dos indicadores econômicos, estimados por animal

\begin{tabular}{|c|c|c|}
\hline Indicador $^{1}$ & Unidade & Equação \\
\hline Custo fixo (CF) & $\mathrm{R} \$$ & $\begin{array}{l}\text { = depreciação (instalações, máquinas, implementos e equipamentos) + } \\
\text { oportunidade (instalações, máquinas, implementos e equipamentos) }+ \\
\text { oportunidade da terra }\end{array}$ \\
\hline Custo variável (CV) & $\mathrm{R} \$$ & $\begin{array}{l}=\text { compra do animal magro }+ \text { controle sanitário, alimentação com } \\
\text { volumoso e concentrado }+ \text { mão-de-obra contratada/diarista } \\
\text { assistência técnica }+ \text { outras despesas operacionais }+ \text { oportunidade do } \\
\text { capital investido }\end{array}$ \\
\hline $\begin{array}{l}\text { Custo operacional } \\
\text { efetivo (COE) }\end{array}$ & $\mathrm{R} \$$ & $\begin{array}{l}=\text { compra do animal magro }+ \text { controle sanitário, alimentação com } \\
\text { volumoso e concentrado }+ \text { mão-de-obra contratada/diarista e } \\
\text { assistência técnica }+ \text { outras despesas operacionais }\end{array}$ \\
\hline $\begin{array}{l}\text { Custo operacional total } \\
\text { (COT) }\end{array}$ & $\mathrm{R} \$$ & $\begin{array}{l}=\mathrm{COE}+\text { depreciação (instalações, máquinas, implementos e } \\
\text { equipamentos) }\end{array}$ \\
\hline Custo total & $\mathrm{R} \$$ & $=(\mathrm{CF}+\mathrm{CV})$ ou $(\mathrm{COT}+$ Oportunidades $)$ \\
\hline Custo/kg ganho de peso & $\mathrm{R} \$ / \mathrm{kg}$ & $\begin{array}{l}=(\text { custo total-custo de compra do animal magro)/ganho de peso total } \\
\text { na terminação }\end{array}$ \\
\hline $\begin{array}{l}\text { Receita com venda do } \\
\text { animal gordo (RANI) }\end{array}$ & $\mathrm{R} \$$ & $=$ peso vivo final $*(\mathrm{R} \$ / \mathrm{kg}$ vivo animal gordo $)$ \\
\hline $\begin{array}{l}\text { Receita com venda de } \\
\text { esterco }(\text { REST) })^{2}\end{array}$ & $\mathrm{R} \$$ & $\begin{array}{l}=\text { peso vivo médio } * 6 \% * \text { dias alimentação } * 31,2 \% \mathrm{MS} * \mathrm{R} \$ \\
0,17661 / \mathrm{kg} \text { MS esterco }\end{array}$ \\
\hline Receita total (RT) & $\mathrm{R} \$$ & $=$ RANI + REST \\
\hline
\end{tabular}

${ }^{1}$ Considerando valores dos anos de 2004 a 2012 deflacionados para 2012.

${ }^{2}$ Conforme Rodrigues Filho et al. ${ }^{14}$ e Ferreira et al. ${ }^{15}$. 
Tabela 2 - Descrição dos indicadores econômicos, estimados por animal

\begin{tabular}{|c|c|c|}
\hline Indicador $^{1}$ & Unidade & Equação \\
\hline Margem bruta (MB) & $\mathrm{R} \$$ & $=\mathrm{RT}-\mathrm{COE}$ \\
\hline Margem líquida (ML) & $\mathrm{R} \$$ & $=\mathrm{RT}-\mathrm{COT}$ \\
\hline Lucro & $\mathrm{R} \$$ & $=\mathrm{RT}-$ custo total \\
\hline Custo/kg ganho de peso & $\mathrm{R} \$ / \mathrm{kg}$ & $\begin{array}{l}=\text { (custo total }- \text { custo de compra do animal magro)/ganho } \\
\text { de peso total na terminação }\end{array}$ \\
\hline Valor presente líquido (VPL) & $\mathrm{R} \$$ & $\begin{array}{l}\sum_{=}^{n} \frac{\text { valores }_{\tilde{i}}}{(1+T M A)^{i}} \text {, onde n=número de fluxos de caixa, } \\
\text { valores=fluxos de caixa e TMA=taxa mínima de } \\
\text { atratividade }\end{array}$ \\
\hline $\begin{array}{l}\text { Índice Benefício: Custo (IB:C), ou } \\
\text { índice de lucratividade }\end{array}$ & & $\begin{array}{l}=\text { Valor presente dos fluxos de caixa positivos/valor } \\
\text { presente dos fluxos de caixa negativos }\end{array}$ \\
\hline $\begin{array}{l}\text { Retorno adicional sobre o } \\
\text { investimento (ROIA) } \\
\text { econômico agregado }\end{array}$ & $\%$ a.m. & $\begin{array}{l}i=\sqrt{\frac{F V}{P V}}-1 \\
=\sqrt{\mathrm{IB}} \mathrm{C}, P V=\text { Valor Presente, } n=\text { Período e } i=\text { Taxa de Juros, } \\
\text { representa o ROIA }\end{array}$ \\
\hline Taxa Interna de Retorno (TIR) & $\%$ a.m. & $\begin{array}{l}-I+\sum_{t=1}^{n} \frac{F C_{t}}{(1+T I R)^{t}} \text {, onde: } I=\text { investimento de capital na } \\
\text { data zero, } F C_{t}=\text { retorno na data } t \text { do fluxo de caixa e } n= \\
\text { prazo de análise do projeto }\end{array}$ \\
\hline $\begin{array}{l}\text { Período de recuperação do } \\
\text { investimento } \\
\text { descontado }(\mathrm{PBd})\end{array}$ & Mês & $\begin{array}{l}=((-1 * \text { valor presente dos fluxos de caixa negativos }) / \text { valor } \\
\text { presente dos fluxos de caixa positivos }) * \text { número de fluxos } \\
\text { de caixa }\end{array}$ \\
\hline
\end{tabular}

${ }^{1}$ Considerando valores dos anos de 2004 a 2012 deflacionados para 2012.

Para as estimativas dos custos com mão-deobra, considerou-se a necessidade de 1 (um) homem/500 bois confinados para a mão-de-obra contratada/diarista (R $\$$ 30,52 homem dia $^{-1}$ ) e dois salários mínimos/mês/1.000 animais para a mão-deobra e assistência técnica (salário mínimo de $\mathrm{R} \$$ 520,11). Para fins de cálculo dos custos com mão-deobra, foram acrescentados dois meses ao período de alimentação, para preparo/manutenção das instalações e demais atividades.

Outras despesas operacionais como manutenção de instalações, máquinas, implementos e equipamentos, combustível, energia elétrica, frete, impostos e alimentação da mão-de-obra foram estimadas pelo equivalente a $2,5 \%$ das despesas operacionais citadas anteriormente para estimar o custo de oportunidade do capital investido.

As equações utilizadas para estimativa dos custos, receitas e indicadores econômicos estão apresentadas nas Tabelas 1 e 2.

Para estimativa dos indicadores econômicos VPL, IB:C, ROIA, TIR e PBd, foram elaborados fluxos de caixa com horizonte de planejamento de um ano, conforme sugestão de Resende Filho et al. ${ }^{12}$.
Para esses indicadores não foram incluídos os custos de oportunidade na estimativa do custo total.

\section{RESULTADOS E DISCUSSÃO}

Verificou-se (Tabela 3) que a diferença entre os pesos de abate extremos representou $18 \%$ (495 vs $421 \mathrm{~kg}$ ), enquanto que o período de alimentação variou 67\% (184 vs 110 dias). Essas informações são interessantes, pois tem relação com a viabilidade econômica. Para o produtor, a comercialização de bovinos junto aos abatedouros-frigoríficos deve atender alguns aspectos relacionados com peso de carcaça e grau de acabamento, de acordo com a idade (dentição) dos animais. Assim, menores pesos de carcaça associados com pouco acabamento, resultam em penalização no preço pago ao produtor. $\mathrm{Na}$ raça Charolês, por exemplo, sabe-se de seu excelente potencial para velocidade de ganho de peso, atingindo elevados pesos na fase adulta, ou seja, caracteriza-se como raça de grande porte. Consequentemente, a deposição de gordura ocorre em pesos mais elevados, exigindo, com isso, maiores períodos de alimentação. Para os animais do presente 
estudo, espessura de gordura acima de $3 \mathrm{~mm}$ (mínimo exigido pelos abatedouros-frigoríficos) foram obtidas apenas no peso de abate de $495 \mathrm{~kg}$, confirmando os comentários anteriores.

Tabela 3 - Média para itens componentes dos custos e suas participações relativas de acordo com o peso de abate

\begin{tabular}{|c|c|c|c|c|c|c|}
\hline \multirow{2}{*}{ Itens } & \multicolumn{6}{|c|}{ Peso de abate, $\mathrm{kg}$} \\
\hline & 421 & $\% *$ & 461 & $\% *$ & 495 & $\% *$ \\
\hline Tempo de alimentação, dias & 110 & - & 145 & - & 184 & - \\
\hline Boi magro, $\mathrm{R} \$ / \mathrm{kg}$ vivo & 2,93 & - & 2,93 & - & 2,93 & - \\
\hline Boi gordo, $\mathrm{R} \$ / \mathrm{kg}$ vivo & 2,83 & - & 2,83 & - & 2,83 & - \\
\hline Custos Fixos (CF), R\$ & 30,21 & 1,96 & 31,27 & 1,81 & 32,45 & 1,67 \\
\hline Depreciação, R\$ & 23,40 & 1,52 & 23,40 & 1,33 & 23,40 & 1,21 \\
\hline Oportunidade da estrutura, $\mathrm{R} \$$ & 1,64 & 0,11 & 1,64 & 0,09 & 1,64 & 0,08 \\
\hline Oportunidade terra, $\mathrm{R} \$$ & 5,16 & 0,33 & 6,22 & 0,36 & 7,41 & 0,38 \\
\hline Custos Variáveis (CV), R\$ & $1.507,87$ & 98,0 & $1.695,20$ & 97,6 & $1.906,69$ & 98,3 \\
\hline Compra do animal magro, $\mathrm{R} \$$ & 870,09 & 56,6 & 870,09 & 50,4 & 870,09 & 44,9 \\
\hline Controle sanitário, R\$ & 19,15 & 1,25 & 19,15 & 1,11 & 19,15 & 0,99 \\
\hline Alimentação volumoso, $\mathrm{R} \$$ & 190,10 & 12,3 & 250,59 & 14,5 & 317,99 & 16,4 \\
\hline Alimentação concentrado, R\$ & 311,44 & 20,2 & 410,54 & 23,8 & 520,96 & 26,9 \\
\hline Mão de obra contratada/diarista, $R \$$ & 17,43 & 1,13 & 21,01 & 1,22 & 25,01 & 1,29 \\
\hline Assistência técnica, $\mathrm{R} \$$ & 15,68 & 1,02 & 18,91 & 1,10 & 22,51 & 1,16 \\
\hline Outros, $\mathrm{R} \$$ & 35,60 & 2,31 & 39,76 & 2,30 & 44,39 & 2,29 \\
\hline Oportunidade capital investido, $\mathrm{R} \$$ & 48,38 & 3,15 & 65,16 & 3,77 & 86,60 & 4,47 \\
\hline Custo Total (CT), R\$ & $1.538,08$ & & $1.726,48$ & & $1.939,15$ & \\
\hline Custo/ kg ganho peso, $\mathrm{R} \$$ & 5,47 & & 5,32 & & 5,23 & \\
\hline Custo Operacional Efetivo, R\$ & $1.459,48$ & & $1.630,04$ & & $1.820,09$ & \\
\hline Custo Operacional Total, $\mathrm{R} \$$ & $1.482,89$ & & $1.653,44$ & & $1.843,49$ & \\
\hline
\end{tabular}

*em relação ao custo total.

Avaliando-se a separação do custo total em fixos e variáveis, notou-se pouca mudança na participação percentual entre os pesos de abate. Em relação aos custos variáveis, o que ocorreram foram alterações na participação percentual de alguns itens que o compõem, principalmente com a fração volumosa, fração concentrada e oportunidade do capital investido, na ordem de 67, 67 e 79\%, respectivamente, passando dos 421 para $495 \mathrm{~kg}$ de peso de abate.

Em relação ao componente alimentação, em sistemas de terminação em confinamento, desconsiderando-se o custo com compra do animal magro, os maiores gastos são decorrentes da alimentação dos animais e, entre os componentes da alimentação, o concentrado é o mais oneroso. Por esse motivo, Pacheco et al. ${ }^{4}$ e Restle et al..$^{5}$ sugerem que uma maneira de reduzir o custo da dieta seria implementar estratégias de comercialização, buscando preços diferenciados em função da época do ano e/ou quantidade adquirida para os itens do concentrado e/ou, conforme Ferreira et al. ${ }^{15}$, pela produção de volumoso a baixo custo visando a viabilidade econômica do confinamento.

A relação entre indicadores de eficiência biológica e econômica é uma maneira direta e fácil de compreender a viabilidade de um investimento, sendo muito relevante na área zootécnica, pois nem sempre a melhor resposta biológica representa o melhor resultado do ponto de vista econômico. Assim, a relação custo/kg ganho de peso, facilmente compreendida pelos técnicos/produtores, vem sendo utilizada com muita frequência em estudos que avaliaram a viabilidade econômica de sistemas de produção de bovinos de corte. Verificou-se (Tabela 3) que o custo/kg ganho de peso apresentou leve redução com o aumento no peso de abate; no entanto, muito acima do preço médio do boi gordo praticado no estado do Rio Grande do Sul, considerando-se a média dos anos de 2004 a 2012. 
Diante dessa situação, Rodrigues Filho et al. ${ }^{14}$ sugerem a necessidade de se buscarem alternativas de mercado que valorizem a qualidade do produto oriundo deste sistema de produção.

Analisando a terminação em confinamento de diferentes categorias (jovem vs superjovem) de diferentes grupos raciais entre as raças Charolês $\mathrm{x}$ Nelore, Pacheco et al. ${ }^{4}$ verificaram que o custo por $\mathrm{kg}$ de ganho de peso foi semelhante entre novilhos jovens e superjovens com similar peso final e menor nos novilhos superjovens alimentados até atingirem estado corporal final similar aos jovens. Em estudos comparando diferentes pesos de abate, Restle et al. ${ }^{5}$ verificaram aumento linear no custo/kg ganho de peso com o aumento no peso de abate (340, 370, 400 ou $430 \mathrm{~kg}$ ) de novilhos superjovens Red Angus, devido à redução no ganho de peso médio diário. Vale ressaltar que o incremento no peso de abate está diretamente relacionado com o tempo de alimentação, refletindo no custo total e, por consequência, nos demais indicadores de viabilidade econômica.

Em relação aos itens com receitas (Tabela 4), notou-se maior participação percentual da receita com a venda do animal gordo; no entanto, a receita com venda de esterco aumentou $48 \%$ quando o peso de abate passou de 421 para $495 \mathrm{~kg}$. No estudo de Rodrigues Filho et al. ${ }^{14}$, avaliando a viabilidade econômica do confinamento de novilhos de origem leiteira, alimentados com diferentes níveis de concentrado e de cama de frango, os autores verificaram que a comercialização do esterco incorporou valores à renda bruta da ordem de 3,0; 3,$7 ; 1,6$; e 2,4\% para os níveis de concentrado na dieta de 50:0, 35:15, 75:0 e 52,5:22,5\%, respectivamente.

Tabela 4 - Média para itens componentes das receitas e suas participações relativas de acordo com o peso de abate

\begin{tabular}{lllllll}
\hline \multirow{2}{*}{ Itens } & \multicolumn{2}{l}{ Peso de abate, $\mathrm{kg}$} \\
\cline { 2 - 7 } & 421 & $\%^{*}$ & 461 & $\%^{*}$ & 495 & $\%^{*}$ \\
\hline Receita total (RT), R\$ & $1.323,78$ & & $1.488,30$ & & $1.643,89$ & \\
$\quad$ Venda animal gordo, $\mathrm{R} \$$ & $1.193,18$ & 90,13 & $1.306,55$ & 87,78 & $1.402,91$ & 85,34 \\
$\quad$ Venda de esterco, R\$ & 130,60 & 9,86 & 181,75 & 12,21 & 240,98 & 14,65 \\
\hline *em relação à receita total. & & & & &
\end{tabular}

Dos indicadores financeiros apresentados na Tabela 5, talvez os mais usuais em sistemas de produção animal, difundidos através de recursos de ensino, pesquisa e extensão, sejam a margem bruta, margem líquida e lucro. Analisando esses três indicadores, notou-se que os valores estimados foram negativos em todos os pesos de abate, aumentando a inviabilidade conforme o aumento nos pesos. Esses resultados estão associados com as diferenças entre as cotações do boi magro e boi gordo, inferior para a última. Conforme explanações de Simões et al. ${ }^{16}$, cada um desses indicadores permitem interpretações distintas. Assim, a margem bruta indica a possibilidade de viabilidade econômica no curto prazo (se for positiva), pois cobre o custo operacional efetivo-COE (desembolsos diretos). Para a margem líquida, valores positivos indicam viabilidade econômica no médio prazo, tendo a possibilidade de se manter no longo prazo, por cobrir o custo operacional total (COE+depreciações), ou seja, permite a formação de capital suficiente para repor os bens de produção no final de suas respectivas vidas úteis. Enquanto que o lucro, se for positivo, demonstra que o investimento foi mais atrativo que outra atividade que remunere o capital em 7,25\% ao ano.

Analisando a terminação de novilhos superjovens terminados em confinamento, RESTLE et al. ${ }^{5}$ verificaram lucro em todos os pesos de abate avaliados, porém com redução linear conforme aumentaram os pesos $(340,370,400$ e $430 \mathrm{~kg})$.

Quanto ao VPL, seu método de estimativa é considerado a técnica robusta de análise de investimentos mais conhecida e utilizada e, como o próprio nome indica, é a concentração de todos os valores esperados de um fluxo de caixa na data zero, utilizando-se a taxa mínima de atratividade ${ }^{7}$. Apresenta vantagens e desvantagens, conforme descrito por Lapponi ${ }^{13}$, e na tomada de decisão, VPL maior que zero é uma condição necessária de aceitação individual do projeto, porém, um projeto de simples substituição com VPL igual a zero pode ser aceito, por exemplo, para garantir que a empresa continue competitiva no mercado. No presente estudo, em todos os pesos de abate que foram considerados projetos de investimento distintos, o 
VPL estimado foi negativo, indicando que o custo inicial não é recuperado nem remunerado de forma completa com a taxa mínima de atratividade, destruindo o valor da empresa, medido na data inicial pelo VPL negativo ${ }^{13}$.

Tabela 5 - Médias para indicadores financeiros estimados por animal de acordo com o peso de abate

\begin{tabular}{|c|c|c|c|}
\hline \multirow{2}{*}{ Indicadores financeiros } & \multicolumn{3}{|c|}{ Peso de abate, $\mathrm{kg}$} \\
\hline & 421 & 461 & 495 \\
\hline $\mathrm{TMA}^{1}, \%$ a.m. & 0,58 & 0,58 & 0,58 \\
\hline $\mathrm{N}^{\mathrm{o}}$ períodos ${ }^{2}$, meses & 6 & 7 & 8 \\
\hline Margem bruta, R\$ & $-266,30$ & $-323,49$ & $-417,18$ \\
\hline Margem líquida, $\mathrm{R} \$$ & $-289,70$ & $-346,90$ & $-440,59$ \\
\hline Lucro, R\$ & $-344,89$ & $-419,93$ & $-536,24$ \\
\hline Valor Presente Líquido, $\mathrm{R} \$$ & $-316,78$ & $-381,92$ & $-483,67$ \\
\hline Índice benefício:custo & 0,85 & 0,83 & 0,81 \\
\hline Retorno Adicional sobre Investimento, \% a.m. & $-2,65$ & $-2,52$ & $-2,58$ \\
\hline Taxa interna de retorno, \% a.m. & $-5,02$ & $-4,57$ & $-4,56$ \\
\hline Payback descontado (PBd), meses & 7,05 & 8,37 & 9,86 \\
\hline
\end{tabular}

${ }^{1}$ Taxa Mínima de Atratividade

${ }^{2}$ Utilizado no Fluxo de caixa

O IB:C representa uma medida de quanto se espera ganhar por unidade de capital investido. A hipótese implícita no cálculo do IB:C é que os recursos liberados ao longo da vida útil do projeto sejam reinvestidos à taxa mínima de atratividade ${ }^{7}$; portanto, IB:C maior que 1 indica que o projeto de investimento é viável. No presente estudo, os IB:C foram menores que $1 \mathrm{em}$ todos os pesos de abate, indicando que a cada $\mathrm{R} \$ 1,00$ investido, retornaria em média, R\$ 0,83, inviabilizando, portanto, a terminação de bovinos em confinamento. Esse resultado é explicado pelo fato de o valor presente dos fluxos de caixa positivos ser menor do que o valor presente dos fluxos de caixa negativos.

O ROIA é considerado por Souza et al. ${ }^{7}$ a melhor estimativa de rentabilidade para um projeto de investimento. Representa, em termos percentuais, a riqueza gerada pelo projeto, e deriva da taxa equivalente ao IB:C para cada período do projeto. Os valores estimados do ROIA no presente estudo foram negativos, com média de $-2,58 \%$.

Analisando a TIR, que por definição é a taxa que torna o VPL de um fluxo de caixa igual a zero ${ }^{7}$, a tomada de decisão utilizando-se esse indicador deve se basear na comparação com a TMA ${ }^{13}$. Se a TIR for maior que a TMA, o projeto é viável, sendo o custo inicial recuperado e remunerado com a TMA e o projeto criará valor não determinado. No presente estudo, a TMA considerada foi de $0,58 \%$ a.m., e em nenhum dos pesos de abate estudados a TIR foi superior a este valor. Demais estudos avaliando diferentes condições sexuais e dietas ${ }^{17,18}$ de bovinos em confinamento, demonstraram resultados variados, no entanto, com tratamentos apresentando TIR superior às principais aplicações disponíveis no mercado financeiro.

Quanto ao indicador financeiro $\mathrm{PBd}$, em contextos dinâmicos, como o de economias globalizadas, esse indicador assume importância no processo de decisões de investimentos, pois, como a tendência é a de mudanças continuas e acentuadas na economia, não se pode esperar muito para recuperar o capital investido sob pena de se alijar das próximas oportunidades de investimentos ${ }^{7}$. É considerado pelos autores citados anteriormente como sendo indicador de risco de projetos de investimentos, pois à medida que o $\mathrm{PBd}$ se aproxima do final do horizonte de planejamento, maior é o risco de inviabilidade do investimento. Para o sistema de confinamento, espera-se PBd coincidentes com o número total de meses previstos para o início e final do período de terminação, pois somente no final da execução do período de confinamento até atingir os pesos de abate estipulados é que se obtêm fluxo de caixa positivo, devido à comercialização dos animais gordos. Observou-se (Tabela 5) que os valores estimados para PBd foram além do número de períodos utilizados no fluxo de caixa para todos os pesos de abate, ou seja, os investimentos apresentaram inviabilidade econômica. 


\section{CONCLUSÕES}

Os custos variáveis foram os mais representativos em relação ao custo total, sendo os itens compra do animal magro e alimentação volumoso e concentrado, os mais relevantes. Os indicadores financeiros apresentaram concordância quanto aos resultados nos diferentes pesos de abate. Apesar dos mesmos indicarem inviabilidade da terminação em confinamento, menores pesos resultaram em menores perdas econômicas. Com base na dieta e categoria animal utilizadas, o confinamento como sistema de terminação utilizado no Rio Grande do Sul representa tecnologia de elevado risco econômico.

\section{REFERÊNCIAS}

1.Millen DD, Pacheco RDL, Arrigoni MDB, Galyean ML, Vasconcelos, JT. A snapshop of management practices for nutritional recommendations used by feedlot nutritionists in Brazil. Journal of Animal Science. 2009;87(10):34273439 .

2.Lopes MA, Magalhães GP. Análise da rentabilidade da terminação de bovinos de corte em condições de confinamento: um estudo de caso. Arquivo Brasileiro de Medicina Veterinária e Zootecnia. 2005;57(3):374-379.

3.Costa EC, Restle J, Vaz FN, Alves Filho DC, Bernardes RALC, Kuss F. Características da carcaça de novilhos Red Angus superprecoces abatidos com diferentes pesos. Revista Brasileira de Zootecnia. 2002;31(1):119-128.

4.Pacheco PS, Restle J, Vaz FN, Freitas AK, Padua JT, Neumann M, Arboitte MZ. Avaliação econômica da terminação em confinamento de novilhos jovens e superjovens de diferentes grupos genéticos. Revista Brasileira de Zootecnia. 2006;35(1):309-320.

5.Restle J, Pacheco PS, Costa EC, Freitas AK, Vaz FN, Brondani IL, Fernandes JJR. Apreciação econômica da terminação em confinamento de novilhos Red Angus superjovens abatidos com diferentes pesos. Revista Brasileira de Zootecnia. 2007;36(4):978-986.

6.Missio RL, Brondani IL, Freitas LS, Sachet RH, Silva JHS, Restle J. Desempenho e avaliação econômica da terminação de tourinhos em confinamento alimentados com diferentes níveis de concentrado na dieta. Revista Brasileira de Zootecnia. 2009;38(7):1309-1316.

7. Souza A, Pedro JJ, Silva WV, Duclós LC. Custos de produção, expectativas de retorno e de risco para o agronegócio do milho na região do Planalto Norte Catarinense / Brasil. Custos e @gronegócio on line. 2010;6(1):140-159.
8.Kreuz CL, Souza A, Schuck E, Cunha SK. Custos de produção, expectativas de retorno e de riscos do agronegócio uva na região dos campos de palmas. Revista Alcance - UNIVALI. 2004;11(2):239-258.

9.Kreuz CL, Souza A. Custos de Produção, Expectativas de Retorno e de Risco do Agronegócio do Alho no Sul do Brasil. ABCustos Associação Brasileira de Custos. 2006;1(1):1-19.

10.Kreuz CL, Souza A, Clemente A. Custos de produção, expectativas de retorno e de riscos do agronegócio mel no planalto norte de Santa Catarina. Custos e @ gronegócio on line. 2008;4(1):46-61.

11.Matsunaga M, Bemelmans PF, Toledo PEN, Dulley RD, Okawa H, Pedroso IA. Metodologia de custo utilizada pelo IEA. Agricultura em São Paulo. 1976;23(1):123-139.

12.Resende Filho MA, Braga MJ, Rodrigues RV. Sistemas de terminação em confinamento: perspectivas para dinamização da cadeia produtiva da carne bovina em Minas Gerais. Revista Brasileira de Economia. 2001;55(1):107-131.

13.Lapponi JC. Projetos de investimento na empresa. 1st ed. Rio de Janeiro: Elsevier; 2007. 488p. Portuguese.

14.Rodrigues Filho M, Mancio AB, Gomes ST, Silva FF, Lana RP, Rodrigues NEB, Soares CA, Veloso CM. Avaliação econômica do confinamento de novilhos de origem leiteira, alimentados com diferentes níveis de concentrado e de cama de frango. Revista Brasileira de Zootecnia. 2002;31(5):2055-2069.

15.Ferreira IC, Silva MA, Barbosa FA, Carvalho ADF, Correa GSS, Fridrich AB, Souza JER. Avaliação técnica e econômica de diferentes grupos genéticos de bovinos de corte machos superprecoces e do sistema de produção em confinamento. Arquivo Brasileiro de Medicina Veterinária e Zootecnia. 2009;61(1):243-250.

16.Simões ARP, Moura AD, Rocha DT. Avaliação econômica comparativa de sistemas de produção de gado de corte sob condições de risco no Mato Grosso do Sul. Revista de Economia e Agronegócio. 2006;5(1):51-72.

17.Fernandes ARM, Sampaio AAM, Henrique W, Perecin D, Oliveira EA, Túllio RR. Avaliação econômica e desempenho de machos e fêmeas Canchim em confinamento alimentados com dietas à base de silagem de milho e concentrado ou cana-de-açúcar e concentrado contendo grãos de girassol. Revista Brasileira de Zootecnia. 2007;36(4):855-864.

18.Coan RM, Reis RA, Resende FD, Sampaio RL, Schocken-Iturrino RP, Garcia GR, Berchielli TT. Viabilidade econômica, desempenho e características de carcaça de garrotes em confinamento alimentados com dietas contendo silagem de capins tanzânia ou marandu ou silagem de milho. Revista Brasileira de Zootecnia. 2008;37(2):311-318 